\title{
Vorwort \\ zur deutschen Übersetzung der vierten Auflage
}

Die TNM-Klassifikation maligner Tumoren ist zuletzt 1978 in 3. Auflage erschienen. Seither haben sich in der Diagnostik durch die weite Verwendung der Computertomographie und durch die Einführung der endoskopischen Sonographie und der Kernspintomographie beträchtliche Fortschritte ergeben; darüber hinaus wurden in vielen Studien neue Erkenntnisse über die Beziehungen zwischen der Tumorausbreitung zum Zeitpunkt der Diagnose und dem weiteren Krankheitsverlauf gewonnen.

Wie festzustellen ist, führten manche Benutzer im Verlauf der Jahre Abwandlungen der Klassifikationsregeln bei verschiedenen anatomischen Bezirken ein. Um diese Entwicklung eigenständiger Modifizierung - eine Einschränkung der Standardisierung - auszugleichen und um die wissenschaftlichen Fortschritte der letzten 10 Jahre zu berücksichtigen, vereinbarten die Nationalen TNM-Komitees, die TNM-Klassifikation von $1978 \mathrm{zu}$ überarbeiten und auch neue Klassifikationen für bisher im TNM-System noch nicht berücksichtigte Organe einzufügen. Besonderes Anliegen sollte es sein, wieder allgemein anerkannte, international einheitliche Regeln zu schaffen.

Die nun vorliegende 4.Auflage der TNM-Klassifikation stimmt mit der Klassifikation gynäkologischer Tumoren durch die FIGO (Fédération Internationale de Gynécologie et d'Obstétrique) überein und ist identisch mit der Klassifikation kindlicher Tumoren durch die SIOP (Société Internationale d'Oncologie Pédiatrique). Sie wurde von allen Nationalen TNM-Komitees einschließlich des AJCC (American Joint Committee on Cancer) angenommen, so daß nunmehr eine auf den neuesten Stand gebrachte, weltweit einheitliche Klassifikation der Tumorausbreitung zur Verfügung steht. 
Es war für das Deutschsprachige TNM-Komitee (DSK) eine selbstverständliche Aufgabe, die 4. Auflage ins Deutsche zu übersetzen und sie damit auch in deutscher Sprache möglichst rasch allgemein zugänglich zu machen. Die im Vergleich zur 3. Auflage geänderten Definitionen der TNM/pTNM-Klassifikation und der Stadiengruppierungen sind links seitlich durch eine Linie gekennzeichnet.

Im Auftrag des Deutschsprachigen TNM-Komitees
P. Hermanek, Erlangen
O. Scheibe, Stuttgart
B. Spiessl, Basel
G. Wagner, Heidelberg 